\title{
Anisotropic hybrid excitation modes in monolayer and double-layer phosphorene on polar substrates
}

\author{
S. Saberi-Pouya, ${ }^{1,2}$ T. Vazifehshenas,,${ }^{1, *}$ T. Salavati-fard,${ }^{3}$ and M. Farmanbar ${ }^{4}$ \\ ${ }^{1}$ Department of Physics, Shahid Beheshti University, G.C., Evin, Tehran 1983969411, Iran \\ ${ }^{2}$ Department of Physics, University of Antwerp, Groenenborgerlaan 171, B-2020 Antwerpen, Belgium \\ ${ }^{3}$ Department of Physics and Astronomy, University of Delaware, Newark, Deleware 19716, USA \\ ${ }^{4}$ Faculty of Science and Technology and MESA+ Institute for Nanotechnology, University of Twente, \\ P.O. Box 217, 7500 AE Enschede, The Netherlands
}

(Received 15 June 2017; published 1 September 2017)

\begin{abstract}
We investigate the anisotropic hybrid surface optical (SO) phonon-plasmon dispersion relations in monolayer and double-layer phosphorene systems located on the polar substrates, such as $\mathrm{SiO}_{2}, h$ - $\mathrm{BN}$, and $\mathrm{Al}_{2} \mathrm{O}_{3}$. We calculate these hybrid modes by using the dynamical dielectric function in the random phase approximation in which the electron-electron interaction and long-range electric field generated by the substrate SO phonons via Fröhlich interaction are taken into account. In the long-wavelength limit, we obtain some analytical expressions for the hybrid SO phonon-plasmon dispersion relations which agree with those obtained from the loss function. Our results indicate a strong anisotropy in SO phonon-plasmon modes, which are stronger along the light-mass direction in our heterostructures. Furthermore, we find that the type of substrate has a significant effect on the dispersion relations of the coupled modes. Importantly, the hybrid excitations are apparently sensitive to the misalignment and separation between layers in double-layer phosphorene.
\end{abstract}

DOI: 10.1103/PhysRevB.96.115402

\section{INTRODUCTION}

Phosphorene, a monolayer of black phosphorus (BP), has recently attracted special attention among two-dimensional materials (2DMs) due to its unique highly anisotropic electronic and optical properties [1-12]. BP is the most stable allotrope of phosphorus at room temperature and pressure. Few-layer phosphorene can be obtained through a mechanical exfoliation method akin to graphene $[13,14]$. However, unlike graphene the phosphorene layers are not perfectly flat and form a puckered surface due to the $\mathrm{sp}^{3}$ hybridization of $3 \mathrm{~s}$ and $3 p$ atomic orbitals. Also, the band gap of BP is direct and can be tuned from $0.3 \mathrm{eV}$ to the visible part of the spectrum $[15,16]$. In contrast, graphene is gapless, and the transition metal dichalcogenides (TMDs) have an indirect gap in the bulk phase and only monolayer TMDs have a direct gap [17]. Moreover, BP exhibits a strong in-plane anisotropic electrical, optical, and phonon properties, which is absent in graphene and TMDs [18].

In comparison to graphene, phosphorene is chemically reactive and tends to form strong bonds with the surface of substrates which leads to some structural changes [19]. Naturally, chemically stable 2DMs, such as graphene and hexagonal boron nitride $(h$-BN), may be used for protecting fragile and low-chemical-stable 2DMs, such as phosphorene [20].

The properties of substrate often drastically alter the transport behavior of the 2D crystal and the overall characteristics of the device. Recently, phosphorene has been transferred to the $h$-BN substrate $[21,22]$. The interaction between phosphorene and substrate is considered to play a crucial role in the modulation of the electronic properties of phosphorene-based devices [21,23]. In most currently available 2DMs, a sample lies on the top of a polar substrate such as $h-\mathrm{BN}, \mathrm{SiO}_{2}, \mathrm{SiC}$, or

*t-vazifeh@sbu.ac.ir
$\mathrm{Al}_{2} \mathrm{O}_{3}$ [24-30]. In such heterostructures, there are polar optical phonon modes that are localized near the 2DMs-substrate interface and coupled to the electronic excitations such as plasmons through the long-range Fröhlich interaction. As a result, the SO phonon could be considered as the dominant phonon-plasmon coupling source in 2DMs on polar substrates.

The coupling of SO phonon modes to the plasma oscillations of free carriers is known as SO phonon-plasmon coupling. These coupled modes may be observed by the infrared (IR) transmission measurements [31,32]. The SO phonon-plasmon coupling changes the dips in the IR reflectivity spectra from isolated plasmon and SO phonon frequencies to the normal coupled ones $[33,34]$.

The coupled SO phonon-plasmon modes are extensively investigated in 2DMs with isotropic band structure such as graphene [35-38]. It is shown that this phenomenon modifies many-body properties such as plasmon modes $[33,39,40]$ and self energy [41] and can also change the mobility [42,43], effective mass [41,44], scattering rate [37,45], and inelastic lifetime [46] in the carrier transport phenomena. Moreover, it can be considered as a mechanism for tuning the band gap $[26,38,47]$.

In this paper, we model a system of phosphorene on polar insulator substrates as shown in Fig. 1. We go beyond the assumption of independent electron and SO phonon modes and consider the coupled SO phonon-plasmon oscillations. Here, we theoretically study the coupled SO phonon-plasmon modes in monolayer and double-layer phosphorene on polar substrates using perturbation theory. We start from the expression for dynamical dielectric function of the coupled system in the random phase approximation (RPA) level of theory, and develop a general formalism, which includes the effect of anisotropic energy dispersion and rotationally misaligned double-layer system. In such systems having the interaction between the electrons and substrate SO phonons could yield to phonon-mediated electron-electron interaction which creates a 


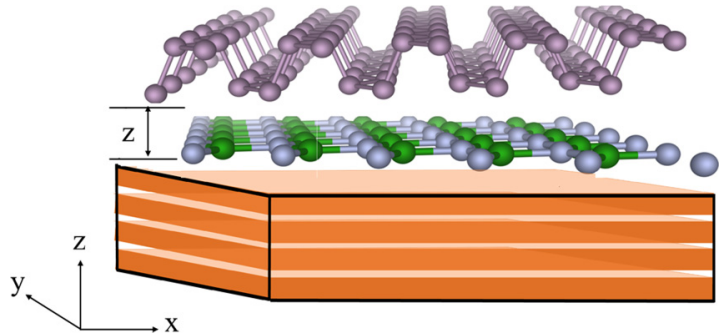

FIG. 1. Schematic figure of a monolayer phosphorene where a polar insulating material (as an example $h$-BN) has been used as a substrate. $z$ indicates the vertical distance of monolayer phosphorene from substrate.

new set of collective modes with highly anisotropic dispersion. We find that in the case of phosphorene in contrast to graphene [33,39] and TMDs [48], the mode coupling effect not only modifies the plasma dispersion relation but also enables us to tune the hybrid SO phonon-plasmon modes in two crystallographic directions. So, the anisotropy is an important feature of the coupled SO phonon-plasmon oscillations spectrum in phosphorene systems.

The paper is organized as follows. In Sec. II we present the theory to calculate the generalized dielectric function in the presence of SO phonon-electron interaction. From the generalized dielectric function, we calculate the coupled SO phonon-plasmon modes in Sec. III and present our results for the coupled SO phonon-plasmon modes in monolayer and double-layer phosphorene. Finally, we summarize the remarks of this paper in Sec. IV.

\section{THEORY}

For an isolated phosphorene system, the anisotropic energy bands in the absence of electron-phonon interaction can be obtained from the $\mathbf{k} \cdot \mathbf{p}$ free electron Hamiltonian:

$$
H_{0}=\left[\begin{array}{cc}
E_{c}+\eta_{c} k_{x}^{2}+v_{c} k_{y}^{2} & \gamma k_{x}+\beta k_{y}^{2} \\
\gamma k_{x}+\beta k_{y}^{2} & E_{v}-\eta_{\nu} k_{x}^{2}-v_{v} k_{y}^{2}
\end{array}\right],
$$

where $E_{c}\left(E_{v}\right)$ is the energy of conduction (valence) band edge, and the effective couplings between the bands are described by $\gamma$ and $\beta$ parameters. The $\eta_{c / v}$ and $v_{c / v}$ are related to the effective masses along $x$ and $y$ directions in the bands. These effective masses can be used to obtain an approximate anisotropic energy dispersion for phosphorene monolayer near the conduction (valence) band minimum (maximum) [15].

In a phosphorene multilayer system with no electronphonon interaction, the electrons in each layer interact with themselves and also with the electrons in other layers through the following electron-electron interaction Hamiltonian:

$$
H_{e l-e l}=\frac{1}{2} \sum_{i j} \sum_{\mathbf{k q p}} v_{i j}(q) a_{\mathbf{k}+\mathbf{q}, i}^{\dagger} a_{\mathbf{p}-\mathbf{q}, j}^{\dagger} a_{\mathbf{p}, j} a_{\mathbf{k}, i},
$$

where $v_{i j}(q)=v(q) e^{-q d_{i j}\left(1-\delta_{i j}\right)}$ represents the diagonal (intralayer with $i=j$ ) and off-diagonal (interlayer with $i \neq j$ ) elements of the bare Coulomb potential matrix. Here, we define $v(q)=2 \pi e^{2} / q \epsilon_{\infty}$ with $\epsilon_{\infty}$ being the high-frequency dielectric constant and $d_{i j}$ is the distance between the $i$ th and $j$ th layers.
Also, $a_{k, i}\left(a_{k, i}^{\dagger}\right)$ is the electron annihilation (creation) operator in layer $i$.

If the phosphorene layers are supported by polar materials, an additional interaction term involving the SO phononplasmon coupling can be included by using Fröhlich Hamiltonian [49]:

$$
H_{e l-p h}=\sum_{i} \sum_{\lambda} \sum_{\mathbf{k q}}\left[M_{0}^{\lambda}(q)\right] a_{\mathbf{k}+\mathbf{q}, i}^{\dagger} a_{\mathbf{k}, i}\left(b_{\mathbf{q} \lambda}+b_{-\mathbf{q} \lambda}^{\dagger}\right),
$$

which renormalizes the screened electron-electron potential through the dielectric function matrix, $\epsilon_{i j}(\omega, \mathbf{q})$ :

$$
V_{i j}^{s c}(\omega, \mathbf{q})=\frac{v_{i j}(q)}{\operatorname{det}\left|\epsilon_{i j}(\omega, \mathbf{q})\right|} .
$$

In Eq. (3), $b_{\mathbf{q} \lambda}\left(b_{\mathbf{q} \lambda}^{\dagger}\right)$ is the phonon annihilation (creation) operator with wave vector $\mathbf{q}$ and branch index $\lambda$ and $M_{0}^{\lambda}(q)$ is the amplitude of the SO phonon-electron interaction $[39,50]$ :

$$
M_{0}^{\lambda}(q)=\left[v(q) \alpha \frac{\omega_{S O}^{\lambda}}{2} e^{-2 q z}\right]^{1 / 2},
$$

where $\omega_{S O}^{\lambda}$ is the SO phonon frequency of the $\lambda$ th branch, $z$ is the vertical distance between phosphorene and the substrate, and we define:

$$
\alpha=\epsilon_{\infty}\left[\frac{1}{\epsilon_{\infty}+1}-\frac{1}{\epsilon_{0}+1}\right],
$$

with $\epsilon_{o}$ being the zero-frequency dielectric constant. Here, we assume that the phonon-phonon interaction is negligible so each mode couples to the electrons, independently.

In order to study the effect of SO phonon-electron coupling on the collective charge-density excitations, one needs to obtain zeros of the determinant of the dynamical dielectric function matrix (the poles of the screened potential). We use the dynamical RPA dielectric function in which the contribution of the SO phonon-electron interaction is taken into account [51]:

$$
\epsilon_{i j}(\omega, \mathbf{q})=\delta_{i j}-U_{i j}(\omega, q) \Pi_{i}(\omega, \mathbf{q}) .
$$

Here, $U_{i j}(\omega, q)$ is the combined Coulomb and phononmediated interactions between $i$ th and $j$ th layers:

$$
U_{i j}(\omega, q)=U_{0}(\omega, q) e^{-q d_{i j}\left(1-\delta_{i j}\right)},
$$

where $U_{0}(\omega, q)=v_{p h}(\omega, q)+v(q)$ and the SO phononmediated electron-electron interaction, $v_{p h}(\omega, q)$, is given by [52]:

$$
v_{p h}(\omega, q)=\sum_{\lambda}\left[M_{0}^{\lambda}(q)\right]^{2} D_{0}^{\lambda}(\omega) .
$$

$D_{0}^{\lambda}(\omega)$ is the bare propagator for a phonon of branch index $\lambda$ :

$$
D_{0}^{\lambda}(\omega)=\frac{2 \omega_{S O}^{\lambda}}{\omega^{2}-\left(\omega_{S O}^{\lambda}\right)^{2}} .
$$

Also, $\Pi_{j}(\omega, \mathbf{q})$ represents the noninteracting dynamic polarization function of layer $j$ which is given by the following expression:

$$
\Pi_{j}(\omega, \mathbf{q})=\frac{g_{s}}{v} \sum_{\mathbf{k}} \frac{f^{j}\left(E_{\mathbf{q}}\right)-f^{j}\left(E_{\mathbf{k}+\mathbf{q}}\right)}{E_{\mathbf{q}}-E_{\mathbf{k}+\mathbf{q}}+\hbar \omega+i \eta},
$$


where $f^{j}\left(E_{\mathbf{q}}\right)$ is the Fermi distribution function of the $j$ th layer at energy $E$ corresponding to the wave vector $\mathbf{q}, g_{s}=2$ is spin degeneracy, and $\eta$ is the broadening parameter which accounts for the disorder in the system. We use the zero-temperature polarization function because this approximation is valid at the typical doping densities $n=5-50 \times 10^{12} \mathrm{~cm}^{-2}$ where the corresponding Fermi temperature $\left(T_{F} \approx 400-800 \mathrm{~K}\right)$ for phosphorene is higher than room temperature. However, the zero-temperature dynamic polarization function for intraband transitions in an anisotropic 2D material can be calculated by making use of the following anisotropic parabolic energy dispersion relation

$$
E_{\mathbf{k}}=\frac{\hbar^{2}}{2}\left(\frac{k_{x}^{2}}{m_{x}}+\frac{k_{y}^{2}}{m_{y}}\right),
$$

in Eq. (11). So one obtains

$$
\begin{aligned}
\frac{\Pi_{i}(\omega, \mathbf{q})}{g_{2 d}}= & \left(\frac{1}{Q_{i}}\right)\left[\left(Z_{-}-\operatorname{sgn}\left(\Re Z_{-}\right) \sqrt{Z_{-}^{2}-1}\right)\right. \\
& \left.-\left(Z_{+}-\operatorname{sgn}\left(\Re Z_{+}\right) \sqrt{Z_{+}^{2}-1}\right)\right] .
\end{aligned}
$$

We define $\mathbf{Q}=\sqrt{m_{d} / \hat{M}}\left(\mathbf{q} / k_{F}\right)$ and $\mathbf{K}=\sqrt{m_{d} / \hat{M}}\left(\mathbf{k} / k_{F}\right)$ where $\hat{M}$ is the mass tensor with diagonal elements $m_{x}$ and $m_{y}$ along $x$ and $y$ directions, $m_{d}=\sqrt{m_{x} m_{y}}, g_{2 d}=m_{d} / \pi \hbar^{2}$ and $Z_{ \pm}=\left((\hbar \omega+i \eta) / \hbar Q k_{F} v_{F}\right) \pm(Q / 2)$ with $v_{F}=\hbar k_{F} / m_{d}$. Вy introducing the rotational angle $\tau_{i}$ as the angle between the $x$ axis in the laboratory frame and $x$ direction of the $i$ th layer, we can write $Q_{i}(\theta)=q \sqrt{m_{d} R_{i}(\theta)} / k_{F}$ in which the orientation parameter $R_{i}(\theta)$ is defined as [6]:

$$
R_{i}(\theta)=\left(\frac{\cos ^{2}\left(\theta-\tau_{i}\right)}{m_{x}}+\frac{\sin ^{2}\left(\theta-\tau_{i}\right)}{m_{y}}\right) .
$$

In the case of monolayer, we will have $\tau_{i}=0$. The knowledge of the appropriate limiting behavior of the polarization function is important in investigating the excitation spectrum especially the collective excitations of the system. Therefore, we obtain the polarization function in the dynamic long-wavelength limit where the plasmon excitations are important due to their long lifetimes. In this limit, the polarization function of Eq. (13) can be approximated as (see Appendix A)

$$
\frac{\Pi_{i}(\omega, q, \theta)}{g_{2 d}} \approx R_{i}(\theta) E_{F} \frac{q^{2}}{\omega^{2}} .
$$

In the following section, we present our calculations for the coupled SO phonon-plasmon modes in both monolayer and double-layer phosphorene systems with a number of experimentally chosen polar substrates/spacers.

\section{RESULTS AND DISCUSSION}

\section{A. Coupled SO phonon-plasmon modes in monolayer phosphorene}

The RPA dielectric function for a monolayer system in which the electrons are coupled to SO phonons of a polar substrate can be obtained through summing over all the bare
TABLE I. Physical parameters of selected polar materials from Ref. [26].

\begin{tabular}{lccc}
\hline \hline & $\mathrm{SiO}_{2}$ & $h-\mathrm{BN}$ & $\mathrm{Al}_{2} \mathrm{O}_{3}$ \\
\hline$\hbar \omega_{S O}^{1}(\mathrm{meV})$ & 60 & 101 & 55 \\
$\hbar \omega_{S O}^{2}(\mathrm{meV})$ & 146 & 195 & 94 \\
$\epsilon_{0}$ & 3.9 & 5.1 & 12.5 \\
$\epsilon_{\infty}$ & 2.5 & 4.1 & 3.2 \\
$\alpha$ & 0.2 & 0.132 & 0.525 \\
\hline \hline
\end{tabular}

bubble diagrams as [39]

$$
\begin{aligned}
\epsilon_{i}(\omega, \mathbf{q})= & 1-\frac{2 \pi e^{2}}{\epsilon_{\infty} q} \Pi_{i}(\omega, \mathbf{q}) \\
& +\sum_{\lambda} \frac{\alpha e^{-2 q z}}{1-\alpha e^{-2 q z}-\omega^{2} /\left(\omega_{s o}^{\lambda}\right)^{2}} .
\end{aligned}
$$

In the long-wavelength limit $(q \rightarrow 0)$ by inserting Eq. (15) into Eq. (16), we get the following coupled collective modes (see Appendix B):

$$
\begin{gathered}
\omega_{(+)}^{\lambda}(q, \theta)=\omega_{s o}^{\lambda}\left(1+\alpha e^{-2 q z} \frac{\omega_{p l}^{2}(q, \theta)}{\left(\omega_{s o}^{\lambda}\right)^{2}}\right), \\
\omega_{(-)}(q, \theta)=\omega_{p l}(q, \theta)\left(1-\frac{\alpha}{2} e^{-2 q z}\right),
\end{gathered}
$$

where $\omega_{p l}(q, \theta)=\sqrt{2 \pi n e^{2} R_{i}(\theta) q / \epsilon_{\infty}}$ is the plasmon frequency of the uncoupled anisotropic system. We consider a phosphorene monolayer sandwiched by polar materials such as $\mathrm{SiO}_{2}, h$-BN, or $\mathrm{Al}_{2} \mathrm{O}_{3}$ which their physical parameters are listed in Table I. In addition, the effective masses in $x$ and $y$ directions of phosphorene monolayer are given as $m_{x} \approx 0.15 m_{0}$ and $m_{y} \approx 0.7 m_{0}$ where $m_{0}$ is the free electron mass [53].

In Fig. 2, we depict the coupled and uncoupled plasmon modes of phosphorene monolayer on $\mathrm{SiO}_{2}$ substrate calculated for two main crystallographic directions $\theta=0(q \| x)$ and $\theta=\pi / 2(q \| y)$. According to this figure in the case of $\mathrm{SiO}_{2}$ as the substrate, there are three hybrid modes, one plasmonlike, $\omega_{-}(q, \theta)$, and two phononlike, $\omega_{+}^{\lambda}(q, \theta)$, as were previously predicted theoretically $[39,41]$ and shown experimentally [31] for graphene monolayer. It can be seen that the $\omega_{-}(q, \theta)$ (plasmonlike mode) is lower in energy than the uncoupled plasmon mode, $\omega_{p l}(q, \theta)$, and the lower (upper) phononlike branch starts from $\omega_{\text {so }}^{1}\left(\omega_{s o}^{2}\right)$ at $q=0$ and increases below (above) the uncoupled plasmon dispersion by increasing $q$ along both directions. The coupling is strong when the frequencies of phononlike modes deviate considerably from the bare SO phonon modes, $\omega_{s o}^{\lambda}$ [see Eq. (17)]. As expected in 2DMs with anisotropic band structure, the hybrid SO phononplasmon modes have higher resonance frequencies [54-56] and the plasmonlike mods are more affected by the SO phononelectron coupling in the direction of the lighter mass, $q \| x$. In the coupled system, the electrons can be scattered either by the emission of hybrid SO phonon-plasmon modes $\left(\omega_{+}, \omega_{-}\right)$ or by the single particle excitation (SPE). The boundary of SPE continuum represented by the green shaded area in Fig. 2 

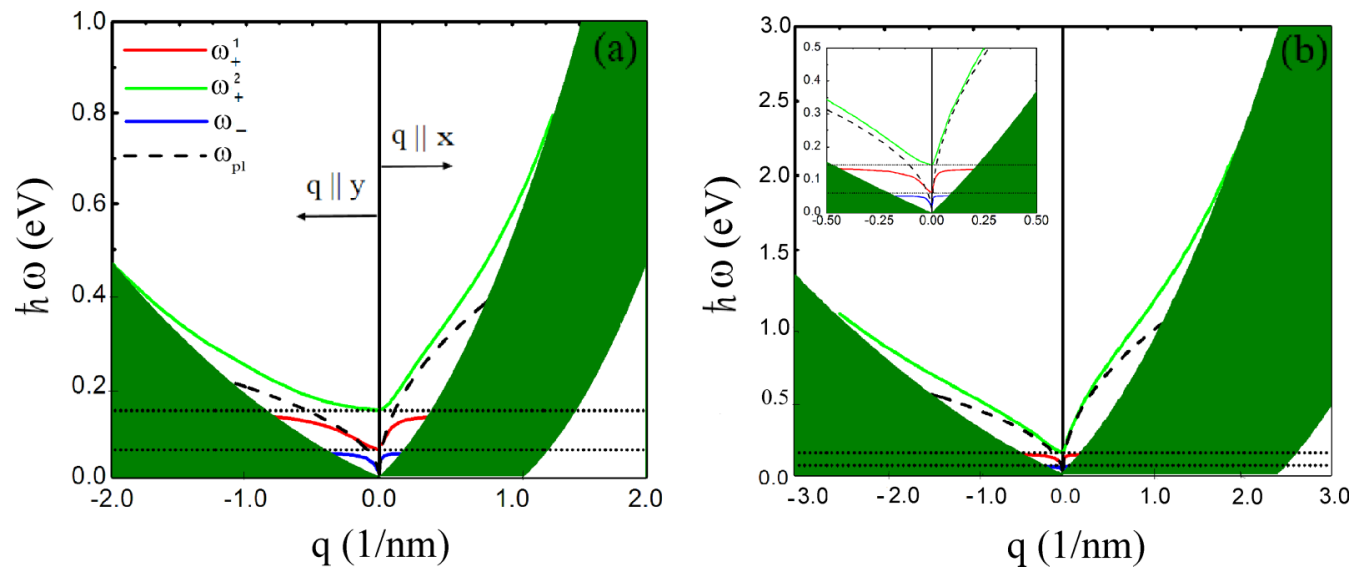

FIG. 2. The hybrid SO phonon-plasmon dispersions in phosphorene monolayer on the $\mathrm{SiO}_{2}$ substrate as a function of wave vector $q$ for two main crystallographic directions of phosphorene: $\theta=0(q \| x)$ and $\theta=\pi / 2(q \| y)$ with (a) $n=1 \times 10^{13} \mathrm{~cm}^{-2},(\mathrm{~b}) n=5 \times 10^{13} \mathrm{~cm}^{-2}$, and $z=0.2 \mathrm{~nm}$. The uncoupled plasmon dispersion is shown by dashed line. The two horizontal lines represent the energies of SO phonon modes, i.e., $\hbar \omega_{s o}^{1}=60 \mathrm{meV}$ and $\hbar \omega_{s o}^{2}=146 \mathrm{meV}$.

is given by:

$$
\hbar \omega_{(S P E)}^{ \pm}(q, \theta)=\frac{R_{i}(\theta) \hbar^{2} q^{2}}{2} \pm \hbar q v_{F} \sqrt{R_{i}(\theta) m_{d}} .
$$

Though all hybrid SO phonon-plasmon excitations are damped at IR frequencies, the plasmonlike mode $\omega_{-}$experiences the Landau damping at smaller $q$ with respect to the phononlike modes, $\omega_{+}^{\lambda}$. On the other hand, the higher frequency phononlike mode, $\omega_{+}^{2}$, stays away from $\omega_{S O}^{2}$ and enters the SPE region at large $q$ for both directions. Therefore, this mode shows a strong coupling and can be easily detected. Moreover, at high densities [Fig. 2(b)] where the Fermi energy exceeds the SO phonon energy, i.e., $E_{F} \gg \hbar \omega_{S O}^{2}$, the upper phononlike branch strongly deviates from the uncoupled SO phonon's energy while the $\omega_{+}^{1}$ branch still remains between the two phonon energies. Thus, the high energy phononlike mode, $\omega_{+}^{2}$, which is very sensitive to the density and direction, can be considered as a tunable quantity for applications in optical plasmonic devices.

Furthermore, we study the impact of angular orientation of $\mathbf{q}$ on the behavior of hybrid SO phonon-plasmon modes by plotting the loss function, $|\Im(1 / \epsilon(\omega, q, \theta))|$, for $q=0.1 \mathrm{~nm}$ (see Fig. 3). The results show that the maximum value of loss function occurs around $\theta=0$ and $\pi$ (along the $x$ direction) for all three branches.

In order to explore the effects of specific substrate on the hybrid SO phonon-plasmon modes, we show the loss functions of phosphorene monolayer on (a) $\mathrm{SiO}_{2}$, (b) $h$-BN, and (c) $\mathrm{Al}_{2} \mathrm{O}_{3}$ polar substrates along $x$ direction $(\theta=0)$ in Fig. 4. One can see that using the $\mathrm{Al}_{2} \mathrm{O}_{3}$ (with higher $\alpha$ ) as a polar substrate results in the strong coupling as evidenced by considerable deviation of $\omega_{-}$from $\omega_{p l}$ and of $\omega_{+}^{\lambda}$ from $\omega_{S O}$.

It should be pointed out that while the phonon frequency $\omega_{S O}$ is an important parameter for the phononlike modes, $\omega_{+}^{\lambda}$, the $\alpha$ parameter mostly affects the plasmonlike spectrum. Hence, the choice of substrate can be used to engineer the plasmon dispersion in phosphorene.

\section{B. Coupled SO phonon-plasmon modes in double-layer phosphorene}

Here, we consider a double-layer phosphorene with equal electron densities sandwiched by a homogeneous dielectric medium that models the substrate. It is reasonable to calculate the uncoupled plasmon modes before discussing the hybrid modes from the zeros of determinant of dielectric function matrix, Eq. (7). In the leading- $q$ approximation (long-wavelength limit), two plasmonic branches are obtained through the following relations [54]:

$$
\begin{gathered}
\omega_{a c}(q, \theta)=2 q \sqrt{\frac{n \pi e^{2} d_{12}}{\epsilon_{\infty}} \frac{R_{1}(\theta) R_{2}(\theta)}{R_{1}(\theta)+R_{2}(\theta)}}, \\
\omega_{o p}(q, \theta)=\sqrt{\frac{2 n \pi e^{2} q}{\epsilon_{\infty}}\left(R_{1}(\theta)+R_{2}(\theta)\right) .}
\end{gathered}
$$

These two uncoupled branches are shown in Fig. 5(a) for $\theta=0$ (along the $x$ direction) and in Fig. 5(b) for $\theta=\pi / 2$ (along the $y$ direction) when $\mathrm{SiO}_{2}$ is considered as a substrate/spacer [54]. It can be seen that the plasmon modes experience a stronger reduction along the $y$ direction compared to the $x$ direction.

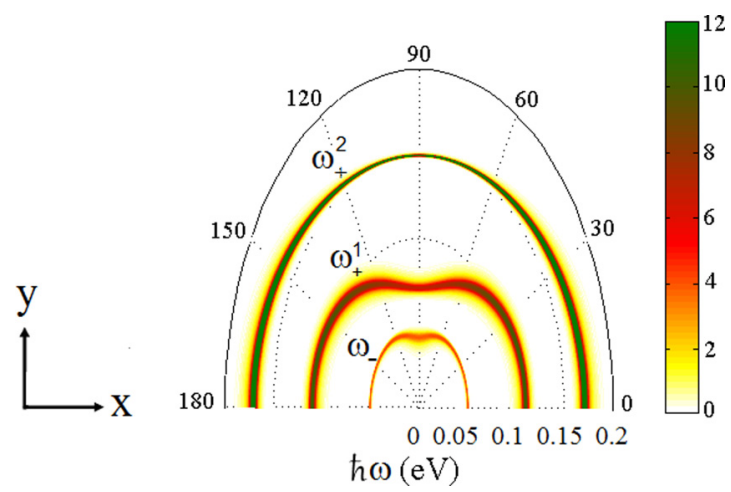

FIG. 3. Loss function, $|\Im(1 / \epsilon(\omega, q, \theta))|$, for $q=0.1 \mathrm{~nm}$ with $n=$ $1 \times 10^{13} \mathrm{~cm}^{-2}$ and $\eta=5 \mathrm{meV}$. The radial and azimuthal coordinates are $\omega$ and the angular orientation of $\mathbf{q}$, respectively. 

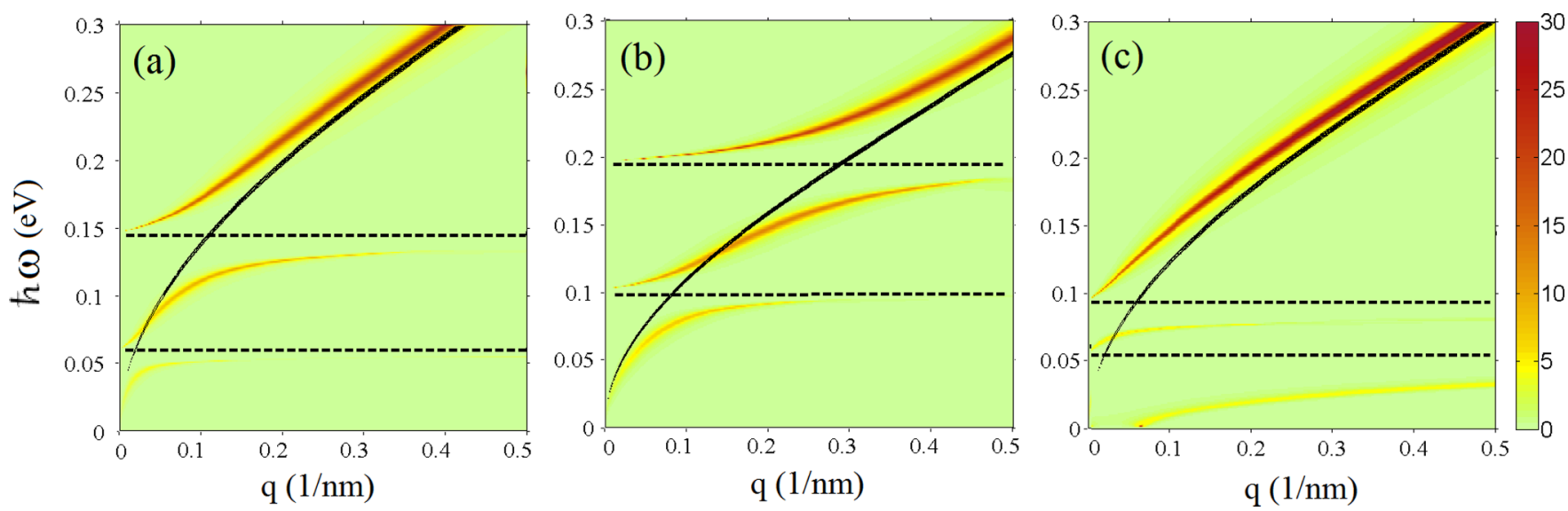

FIG. 4. Effect of changing substrate on the loss function, $|\Im(1 / \epsilon(\omega, q, \theta))|$, for phosphorene monolayer in $(\omega, q)$ space along $\theta=0(q \| x)$. The solid black line is the uncoupled plasmon branch and the two horizontal black dashed lines represent the energies of SO phonon modes of substrate: (a) $\mathrm{SiO}_{2}$, (b) $h$-BN, and (c) $\mathrm{Al}_{2} \mathrm{O}_{3}$ with $n=1 \times 10^{13} \mathrm{~cm}^{-2}, z=0.2 \mathrm{~nm}$ and $\eta=5 \mathrm{meV}$.

For the case of SO phonon-plasmon coupling in a double-layer phosphorene, we find two acoustic phononlike modes, $\omega_{a c(+)}^{\lambda}$, with $\lambda=1,2$ and one acoustic plasmonlike mode, $\omega_{a c(-)}$ (see Appendix B). The long-wavelength dispersions of the acoustic modes can be written as:

$$
\begin{gathered}
\omega_{a c(+)}^{\lambda}(q, \theta)=\omega_{S O}^{\lambda} \sqrt{1+\frac{4 n \pi d_{12} e^{2} q^{2} \alpha e^{-2 q z}}{\left(\omega_{S O}^{\lambda}\right)^{2} \epsilon_{\infty}} \frac{R_{1}(\theta) R_{2}(\theta)}{R_{1}(\theta)+R_{2}(\theta)}}, \\
\omega_{a c(-)}(q, \theta)=2 q \sqrt{\frac{n \pi e^{2} d_{12}\left(1-\alpha e^{-2 q z}\right)}{\epsilon_{\infty}} \frac{R_{1}(\theta) R_{2}(\theta)}{R_{1}(\theta)+R_{2}(\theta)}} .
\end{gathered}
$$
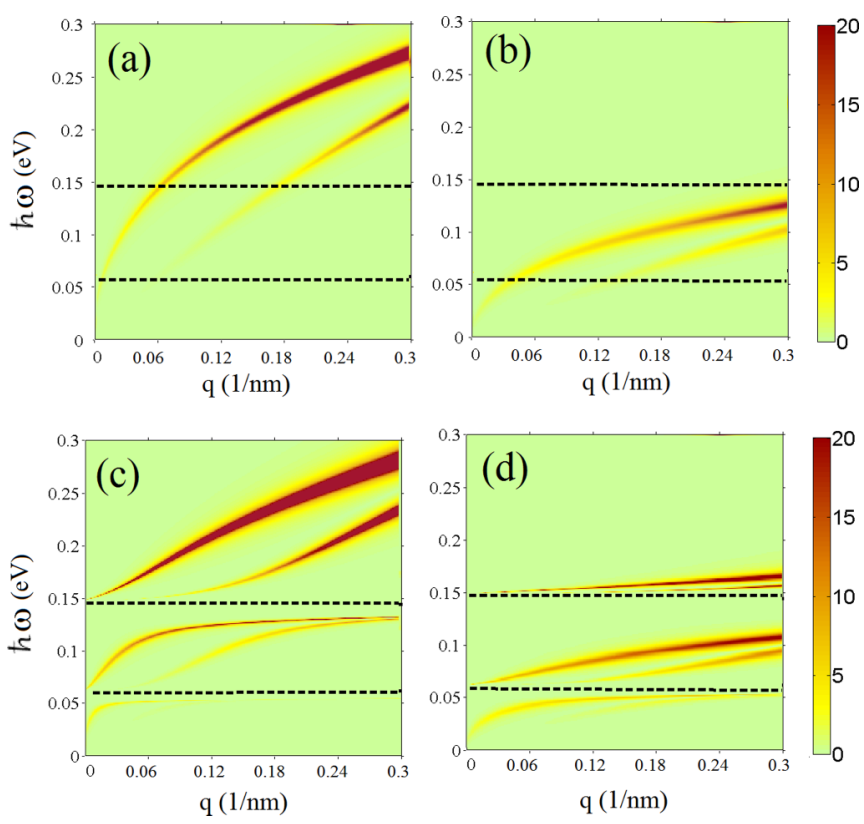

FIG. 5. (a),(b) The uncoupled and (c),(d) coupled SO phononplasmon modes for two aligned phosphorene monolayers sandwiched by $\mathrm{SiO}_{2}$ as a function of wave vector $q$ for two main crystallographic directions: (a),(c) $\theta=0(q \| x)$ and (b),(d) $\theta=\pi / 2(q \| y)$ with $n=1 \times 10^{13} \mathrm{~cm}^{-2}, d_{12}=5 \mathrm{~nm}, z=0.2 \mathrm{~nm}$, and $\eta=5 \mathrm{meV}$.
In the same vein, we obtain two optical phononlike modes $\omega_{o p(+)}^{\lambda}$ and one optical plasmonlike mode $\omega_{o p(-)}$ as

$$
\begin{gathered}
\omega_{o p(+)}^{\lambda}(q, \theta)=\omega_{S O}^{\lambda} \sqrt{1+\frac{2 n \pi e^{2} q \alpha e^{-2 q z}}{\left(\omega_{S O}^{\lambda}\right)^{2} \epsilon_{\infty}}\left(R_{1}(\theta)+R_{2}(\theta)\right)} . \\
\omega_{o p(-)}(q, \theta)=\sqrt{\frac{2 n \pi e^{2} q\left(1-\alpha e^{-2 q z}\right)}{\epsilon_{\infty}}\left(R_{1}(\theta)+R_{2}(\theta)\right)} .
\end{gathered}
$$

The behavior of the coupled modes with $\mathrm{SiO}_{2}$ as substrate/spacer is presented in Figs. 5(c) and 5(d) for $\theta=0$ and $\theta=\pi / 2$, respectively. The dispersion relations of $\omega_{o p / a c(-)}$ modes resemble the uncoupled acoustic and optical plasmon modes with an additional $\left(1-\alpha e^{-2 q z}\right)^{1 / 2}$ multiplier which makes these coupled modes lie lower than the uncoupled ones.

Similar to the case of phosphorene monolayer, the hybrid SO phonon-plasmon frequencies along $q \| x$ are larger than along $q \| y$ because the carriers along the $y$ direction have larger mass and so get damped faster. One can also see that the uncoupled plasmon modes for $q \| y$ lie lower than $\omega_{+}^{2}$ and therefore SO phonon-plasmon coupling along the $y$ direction is substantially weaker compared to the $x$ direction.

In order to understand how the orientation parameter $R_{i}(\theta)$ impacts the behavior of hybrid SO phonon-plasmon modes in the double-layer system, we show three cases in which $\tau_{1}=0$ and $\tau_{2}$ progressively increases from $\pi / 4$ to $\pi / 3$ and to $\pi / 2$ along the $x$ direction and for substrate/spacer $\mathrm{SiO}_{2}$ in Fig. 6 . One may notice that, in general, with the reduction of $\tau_{2}$, the coupled SO phonon-plasmon modes become significantly stronger because the hybrid modes are larger along the lower mass direction, i.e., $\theta=0$. Furthermore, it is clear that the three acoustic plasmonlike/phononlike modes are very sensitive to the rotation of layers and get damped as the angle of rotation is increased. Finally, we address the effect of separation between layers, $d_{12}$, on the coupled modes along the $x$ direction in Fig. 7 for the substrate/spacer $\mathrm{SiO}_{2}$. Here, increasing the separation between layers shows a similar effect on hybrid 

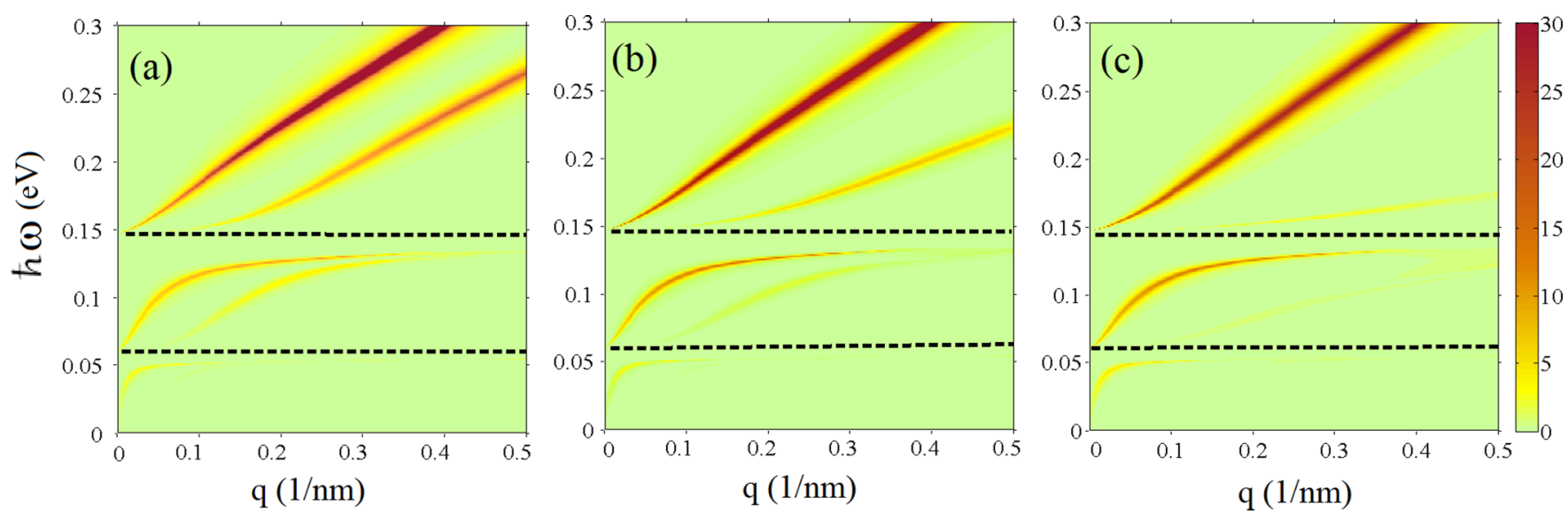

FIG. 6. Effect of changing rotation angle on the loss function, $|\Im[1 / \operatorname{det} \varepsilon(\omega, q, \theta)]|$, for double-layer phosphorene sandwiched by $\mathrm{SiO}_{2}$ in $(\omega, q)$ space along $\theta=0(q \| x)$ for $\tau_{1}=0$ and (a) $\tau_{2}=\pi / 4$, (b) $\tau_{2}=\pi / 3$, and (c) $\tau_{2}=\pi / 2$ with $d_{12}=5 \mathrm{~nm}, n=1 \times 10^{13} \mathrm{~cm}^{-2}$, and $z=0.2 \mathrm{~nm}$.

mode energies as the reduction of rotation angle $\tau_{2}$ (see Fig. 6). As expected, the hybrid acoustic branches, similar to the uncoupled ones, depend strongly on the separation between layers at long wavelengths and move to the optical branches by increasing the layers' spacing. As a result, by adjusting two parameters $\tau_{2}$ and $d_{12}$, the acoustic branches may get strongly damped or a transition to the optical modes may be observed.

\section{CONCLUSION}

In summary, we have considered monolayer and doublelayer phosphorene systems located on commonly used polar substrates $\left(\mathrm{SiO}_{2}, h\right.$-BN, and $\left.\mathrm{Al}_{2} \mathrm{O}_{3}\right)$ and calculated the anisotropic coupled $\mathrm{SO}$ phonon-plasmon dispersion relations. In the presented theory, the dynamical dielectric function is calculated within the RPA which includes the many-body electron-electron interaction in phosphorene layer(s) as well as the interaction between electrons and the long-range electric field generated by the substrate SO phonons. More importantly, we have obtained some analytical expressions for the SO phonon-plasmon dispersion relations in the longwavelength limit which yield the same results of loss function from dielectric function. In the long-wavelength limit, three hybrid SO phonon-plasmon branches are obtained due to two relevant SO phonon modes of polar substrates in monolayer phosphorene. In the case of double layer, these hybrid modes are doubled with three acoustic $\omega_{a c( \pm)}$ and three optical modes $\omega_{o p( \pm)}$. We have shown that these hybrid modes are considerably stronger along the $x$ direction because of lower effective mass of electrons. Moreover, by increasing the electron density, the hybrid excitation modes become stronger at both directions, simultaneously. Among mentioned substrates, we have found that $\mathrm{Al}_{2} \mathrm{O}_{3}$ as a polar substrate leads to more pronounced coupling of phonon-plasmon modes in comparison to $h$ - $\mathrm{BN}$ and $\mathrm{SiO}_{2}$ for the electron density used here. Therefore, the phonon frequency of a substrate, $\omega_{S O}$, is the most effective parameter in phononlike modes, $\omega_{+}^{\lambda}$, whereas $\alpha$ parameter changes the plasmonlike mode, $\omega_{-}$. Hence, the choice of substrate can be utilized in order to
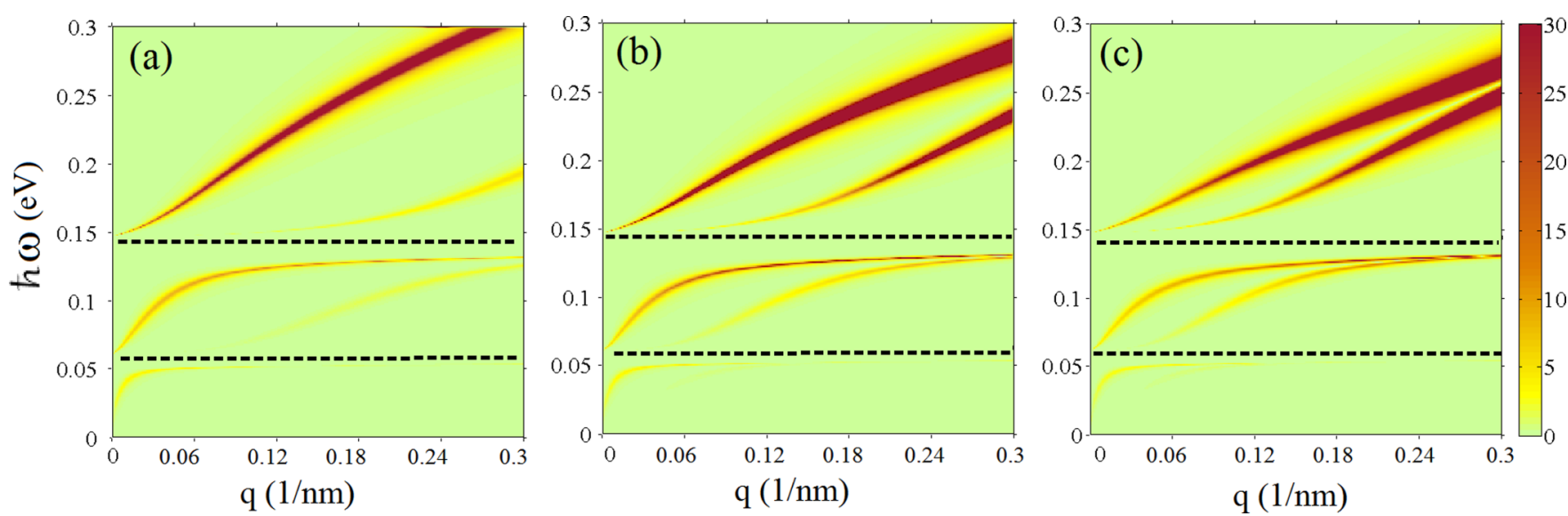

FIG. 7. Effect of changing separation between layers on the loss function, $|\Im[1 / \operatorname{det} \varepsilon(\omega, q, \theta)]|$, for the two parallel phosphorene monolayers sandwiched by $\mathrm{SiO}_{2}$ in $(\omega, q)$ space along $\theta=0(q \| x)$ for (a) $d_{12}=2 \mathrm{~nm}$, (b) $d_{12}=5 \mathrm{~nm}$, and (c) $d_{12}=8 \mathrm{~nm}$ with $n=1 \times 10^{13} \mathrm{~cm}^{-2}$ and $z=0.2 \mathrm{~nm}$. 
engineer the SO phonon-plasmon dispersion in phosphorene. We have also investigated the effect of misalignment of two layers on the hybrid modes in double-layer phosphorene and found that the acoustic phononlike modes are more affected by rotation. In addition, we have observed that by decreasing separation between two layers the acoustic modes move away from the optical modes and quickly damp. As a result, the rotation angle and separation between two layers can be used as a mechanism for tuning the SO phonon-plasmon coupling effects.

\section{APPENDIX A: THE POLARIZATION FUNCTION IN LONG-WAVELENGTH LIMIT}

The dynamic polarization function given by Eq. (11) has the following expression in the long-wavelength limit at zero temperature:

$$
\begin{aligned}
\frac{\Pi(\omega, q, \theta)}{g_{2 d}}= & \frac{1}{2}\left[-2+\sqrt{\left(1-\frac{2 \omega}{k_{F} v_{F} Q^{2}}\right)^{2}-\frac{4}{Q^{2}}}\right. \\
& \left.+\sqrt{\left(1+\frac{2 \omega}{k_{F} v_{F} Q^{2}}\right)^{2}-\frac{4}{Q^{2}}}\right] .
\end{aligned}
$$

One can rewrite Eq. (A1) as:

$$
\begin{aligned}
\frac{\Pi(\omega, q, \theta)}{g_{2 d}}= & \frac{1}{2}\left[-2+\left(1-\frac{2 \omega}{k_{F} v_{F} Q^{2}}\right)\left(1-\frac{2 / Q^{2}}{\left(1-\frac{2 \omega}{k_{F} v_{F} Q^{2}}\right)^{2}}\right)\right. \\
& \left.+\left(1+\frac{2 \omega}{k_{F} v_{F} Q^{2}}\right)\left(1-\frac{2 / Q^{2}}{\left(1+\frac{2 \omega}{k_{F} v_{F} Q^{2}}\right)^{2}}\right)\right]
\end{aligned}
$$

and find the approximate relation:

$$
\frac{\Pi(\omega, q \rightarrow 0, \theta)}{g_{2 d}} \approx \frac{-2}{Q^{2}}\left(\frac{1}{1-\frac{4 \omega^{2}}{\left(k_{F} v_{F} Q^{2}\right)^{2}}}\right) .
$$

Finally, by ignoring unity in the denominator and making use of $Q_{i}(\theta)=q \sqrt{m_{d} R_{i}(\theta)} / k_{F}$ and $E_{F}=\hbar^{2} k_{F}^{2} / 2 m_{d}$, one obtains Eq. (15).

\section{APPENDIX B: SO PHONON-PLASMON MODES IN LONG-WAVELENGTH LIMIT}

\section{Monolayer}

Inserting Eq. (15) into Eq. (16), we have:

$$
\begin{aligned}
\epsilon(\omega, q, \theta)= & 1-\frac{\omega_{p l}^{2}(q, \theta)}{\omega^{2}(q, \theta)} \\
& +\sum_{\lambda} \frac{\alpha e^{-2 q z}}{1-\alpha e^{-2 q z}-\omega^{2}(q, \theta) /\left(\omega_{s o}^{\lambda}\right)^{2}} .
\end{aligned}
$$

From the zeros of dielectric function (here we drop the $q$ and $\theta$ for simplicity):

$$
\begin{gathered}
\omega^{2}\left(\omega^{2}-\left(\omega_{S O}^{\lambda}\right)^{2}\right)-\omega_{p l}^{2}\left(\omega^{2}-\left(\omega_{S O}^{\lambda}\right)^{2}\right) \\
-\omega_{p l}^{2}\left(\omega_{S O}^{\lambda}\right)^{2} \alpha e^{-2 q z}=0,
\end{gathered}
$$

the coupled SO phonon-plasmon modes are obtained as:

$$
\begin{aligned}
\left(\omega_{( \pm)}^{\lambda}\right)^{2}= & \frac{1}{2}\left[\left(\left(\omega_{S O}^{\lambda}\right)^{2}+\omega_{p l}^{2}\right) \pm\left[\left(\left(\omega_{S O}^{\lambda}\right)^{2}-\omega_{p l}^{2}\right)\right.\right. \\
& \left.\left.+4 \omega_{p l}^{2}\left(\omega_{S O}^{\lambda}\right)^{2} \alpha e^{-2 q z}\right]^{1 / 2}\right] .
\end{aligned}
$$

By Taylor expanding the right hand side of Eq. (B3), we get:

$$
\begin{gathered}
\omega_{(+)}^{\lambda}(q, \theta)=\omega_{s o}^{\lambda}\left(1+\alpha e^{-2 q z} \frac{\omega_{p l}^{2}}{\left(\omega_{s o}^{\lambda}\right)^{2}-\omega_{p l}^{2}}\right) \\
\omega_{(-)}^{\lambda}(q, \theta)=\omega_{p l}\left(1-\frac{\alpha e^{-2 q z}}{2} \frac{\left(\omega_{s o}^{\lambda}\right)^{2}}{\left(\omega_{s o}^{\lambda}\right)^{2}-\omega_{p l}^{2}}\right) .
\end{gathered}
$$

Since $\omega_{p l} \rightarrow 0$ at the long-wavelength limit, one can safely ignore $\omega_{p l}$ in the denominator of the above equations and obtain Eqs. (17) and (18).

\section{Double layer}

Here, we calculate the coupled SO phonon-plasmon modes in double-layer systems with anisotropic band structure. In such systems, the rotation of one layer with respect to the other should be considered. In order to determine the dispersion relation of the coupled modes, we need to calculate the zeros of determinant of the total dielectric matrix [Eq. (7)] in the long-wavelength limit:

$$
\begin{aligned}
\epsilon(\omega, q, \theta)= & 1-\left(R_{1}(\theta)+R_{2}(\theta)\right) \Pi^{\prime}(q, \omega) U_{0}(q, \omega) \\
& +\left(U_{0}(q, \omega) \Pi^{\prime}(q, \omega)\right)^{2} R_{1}(\theta) R_{2}(\theta)\left(1-e^{-2 q d_{12}}\right),
\end{aligned}
$$

where $\Pi^{\prime}(\omega, q, \theta)=\Pi_{i}(\omega, q, \theta) / R_{i}(\theta)$. By doing some algebra, we get the following relation:

$$
\begin{aligned}
\epsilon(\omega, q, \theta)= & U_{0}^{2}(q, \omega)\left(2 q d_{12}\right) R_{1}(\theta) R_{2}(\theta) \\
& \times\left[\Pi^{\prime}(\omega, q, \theta)-\Pi_{+}(\omega, q, \theta)\right] \\
& \times\left[\Pi^{\prime}(\omega, q, \theta)-\Pi_{-}(\omega, q, \theta)\right],
\end{aligned}
$$

where $\Pi_{+}$and $\Pi_{-}$defined as

$$
\Pi_{+}(\omega, q, \theta)=\frac{R_{1}(\theta)+R_{2}(\theta)}{\left(R_{1}(\theta) R_{2}(\theta) U_{0}(q, \omega) 2 q d_{12}\right.}
$$

and

$$
\Pi_{-}(\omega, q, \theta)=\frac{1}{\left(R_{1}(\theta)+R_{2}(\theta)\right) U_{0}(q, \omega)} .
$$

The dispersion relation of the couple modes is given by $\Pi^{\prime}(\omega, q, \theta)=\Pi_{ \pm}(\omega, q, \theta)$. Using the Eqs. (5)-(10) in the $\Pi^{\prime}(\omega, q, \theta)=\Pi_{+}(\omega, q, \theta)$ condition, we get:

$$
\begin{aligned}
& \omega^{2}\left(\omega^{2}-\left(\omega_{S O}^{\lambda}\right)^{2}\right) \\
& \quad-q^{2} f_{a c}(\theta)\left[\omega^{2}-\left(\omega_{S O}^{\lambda}\right)^{2}+\left(\omega_{S O}^{\lambda}\right)^{2} \alpha e^{-2 q z}\right]=0,
\end{aligned}
$$

where $f_{a c}(\theta)$ is defined as:

$$
f_{a c}(\theta)=\frac{4 \pi n e^{2} d_{12}}{\epsilon_{\infty}} \frac{R_{1}(\theta) R_{2}(\theta)}{R_{1}(\theta)+R_{2}(\theta)} .
$$


After some algebra, we derive the following relation:

$$
\left(\omega_{a c( \pm)}^{\lambda}\right)^{2}=\frac{\left(\omega_{s o}^{\lambda}\right)^{2}+q^{2} f_{a c}(\theta)}{2} \pm \sqrt{\frac{\left(\left(\omega_{S O}^{\lambda}\right)^{2}-q^{2} f_{a c}(\theta)\right)^{2}+4 q^{2} f_{a c}(\theta)\left(\omega_{S O}^{\lambda}\right)^{2} \alpha e^{-2 q z}}{4}}
$$

which can be simplified in the long-wavelength limit as:

$$
\left(\omega_{a c(+)}^{\lambda}\right)^{2}=\left(\omega_{s o}^{\lambda}\right)^{2}\left[1+\frac{f_{a c}(\theta) q^{2} \alpha e^{-2 q z}}{\left(\omega_{s o}^{\lambda}\right)^{2}}\right]
$$

and

$$
\left(\omega_{a c(-)}^{\lambda}\right)^{2}=f_{a c}(\theta) q^{2}\left[1-\alpha e^{-2 q z}\right] .
$$

Substituting the relation for $f_{a c}(\theta)$ in the above relations, one obtains Eqs. (22) and (23) for the coupled acoustic modes. In the case of the coupled optical modes, we use the $\Pi^{\prime}(q, \omega)=\Pi_{-}(q, \omega)$ condition and get the following equation:

$$
\omega^{2}\left(\omega^{2}-\left(\omega_{s o}^{\lambda}\right)^{2}\right)-f_{o p}(\theta)\left[\omega^{2}-\left(\omega_{s o}^{\lambda}\right)^{2}+\left(\omega_{s o}^{\lambda}\right)^{2} \alpha e^{-2 q z}\right]=0
$$

where $f_{o p}(\theta)$ is given by:

$$
f_{o p}(\theta)=\frac{2 \pi n e^{2}}{\epsilon_{\infty}}\left(R_{1}(\theta)+R_{2}(\theta)\right)
$$

Solving Eq. (B15) yields:

$$
\left(\omega_{o p( \pm)}^{\lambda}\right)^{2}=\frac{\left(\omega_{s o}^{\lambda}\right)^{2}+q f_{o p}(\theta)}{2} \pm \sqrt{\frac{\left(\left(\omega_{s o}^{\lambda}\right)^{2}-q f_{o p}(\theta)\right)^{2}-4 q f_{o p}(\theta)\left(\omega_{s o}^{\lambda}\right)^{2}\left(1-\alpha e^{-2 q z}\right)}{4}}
$$

In the long-wavelength limit, we end up with the following expressions for the coupled optical modes:

$$
\left(\omega_{o p(+)}^{\lambda}\right)^{2}=\left(\omega_{s o}^{\lambda}\right)^{2}\left[1+\frac{q f_{o p}(\theta) \alpha e^{-2 q z}}{\left(\omega_{s o}^{\lambda}\right)^{2}}\right]
$$

and

$$
\left(\omega_{o p(-)}^{\lambda}\right)^{2}=q f_{o p}(\theta)\left[1-\alpha e^{-2 q z}\right] .
$$

Finally, by inserting $f_{o p}(\theta)$ into Eqs. (B18) and (B19) one obtains Eqs. (24) and (25).

[1] D. A. Prishchenko, V. G. Mazurenko, M. I. Katsnelson, and A. N. Rudenko, 2D Materials 4, 025064 (2017).

[2] F. Xia, H. Wang, and Y. Jia, Nat. Commun. 5, 4458 (2014).

[3] T.-H. Liu and C.-C. Chang, Nanoscale 7, 10648 (2015).

[4] Y. Aierken, D. Çakır, C. Sevik, and F. M. Peeters, Phys. Rev. B 92, 081408 (2015).

[5] J. E. Padilha, A. Fazzio, and A. J. R. da Silva, Phys. Rev. Lett. 114, 066803 (2015)

[6] S. Saberi-Pouya, T. Vazifehshenas, M. Farmanbar, and T. Salavati-fard, J. Phys.: Condens. Matter 28, 285301 (2016).

[7] W. Zhu, S. Park, M. N. Yogeesh, K. M. McNicholas, S. R. Bank, and D. Akinwande, Nano Lett. 16, 2301 (2016).

[8] B. Ostahie and A. Aldea, Phys. Rev. B 93, 075408 (2016).

[9] B. Sa, Y.-L. Li, Z. Sun, J. Qi, C. Wen, and B. Wu, Nanotechnology 26, 215205 (2015).

[10] G. C. Constantinescu and N. D. M. Hine, Nano Lett. 16, 2586 (2016).

[11] H. Doh and H. J. Choi, 2D Materials 4, 025071 (2017).

[12] S. Saberi-Pouya, T. Vazifehshenas, T. Salavati-fard, M. Farmanbar, and F. M. Peeters, Phys. Rev. B 96, 075411 (2017).

[13] J. R. Brent, N. Savjani, E. A. Lewis, S. J. Haigh, D. J. Lewis, and P. O'Brien, Chem. Commun. 50, 13338 (2014).
[14] D. Hanlon, C. Backes, E. Doherty, C. S. Cucinotta, N. C. Berner, C. Boland, K. Lee, A. Harvey, P. Lynch, Z. Gholamvand et al., Nat. Commun. 6, 8563 (2015).

[15] A. S. Rodin, A. Carvalho, and A. H. Castro Neto, Phys. Rev. Lett. 112, 176801 (2014).

[16] D. Çakır, H. Sahin, and F. M. Peeters, Phys. Rev. B 90, 205421 (2014).

[17] Q. H. Wang, K. Kalantar-Zadeh, A. Kis, J. N. Coleman, and M. S. Strano, Nat. Nanotechnol. 7, 699 (2012).

[18] A. Carvalho, M. Wang, X. Zhu, A. S. Rodin, H. Su, and A. H. C. Neto, Nat. Rev. Mater. 1, 16061 (2016).

[19] L. Shao, H. Ye, Y. Wu, D. Yinxiao, P. Ding, F. Zeng, and Q. Yuan, Mater. Res. Express 3, 025013 (2016).

[20] M. Bokdam, P. A. Khomyakov, G. Brocks, Z. Zhong, and P. J. Kelly, Nano Lett. 11, 4631 (2011).

[21] R. A. Doganov, S. P. Koenig, Y. Yeo, K. Watanabe, T. Taniguchi, and B. Özyilmaz, Appl. Phys. Lett. 106, 083505 (2015).

[22] M. Moreno-Moreno, G. Lopez-Polin, A. Castellanos-Gomez, C. Gomez-Navarro, and J. Gomez-Herrero, 2D Materials 3, 031007 (2016).

[23] J. Gao, G. Zhang, and Y.-W. Zhang, J. Am. Chem. Soc. 138, 4763 (2016). 
[24] C. R. Dean, A. F. Young, I. Meric, C. Lee, L. Wang, S. Sorgenfrei, K. Watanabe, T. Taniguchi, P. Kim, K. Shepard et al., Nat. Nanotechnol. 5, 722 (2010).

[25] X. Chen, Y. Wu, Z. Wu, Y. Han, S. Xu, L. Wang, W. Ye, T. Han, Y. He, Y. Cai et al., Nat. Commun. 6, 7315 (2015).

[26] A. Mogulkoc, Y. Mogulkoc, A. N. Rudenko, and M. I. Katsnelson, Phys. Rev. B 93, 085417 (2016).

[27] M. Ishigami, J. Chen, W. Cullen, M. Fuhrer, and E. Williams, Nano Lett. 7, 1643 (2007).

[28] S. Dai, Q. Ma, M. Liu, T. Andersen, Z. Fei, M. Goldflam, M. Wagner, K. Watanabe, T. Taniguchi, M. Thiemens et al., Nat. Nanotechnol. 10, 682 (2015).

[29] M. Zarenia, O. Leenaerts, B. Partoens, and F. M. Peeters, Phys. Rev. B 86, 085451 (2012).

[30] M. Buscema, G. A. Steele, H. S. J. van der Zant, and A. Castellanos-Gomez, Nano Research 7, 561 (2014).

[31] H. Yan, T. Low, W. Zhu, Y. Wu, M. Freitag, X. Li, F. Guinea, P. Avouris, and F. Xia, Nat. Photon. 7, 394 (2013).

[32] Z. Liu and K. Aydin, Nano Lett. 9, 312 (2016).

[33] V. W. Brar, M. S. Jang, M. Sherrott, S. Kim, J. J. Lopez, L. B. Kim, M. Choi, and H. Atwater, Nano Lett. 14, 3876 (2014).

[34] M. A. Stroscio and M. Dutta, Phonons in Nanostructures (Cambridge University Press, Cambridge, 2001).

[35] J.-H. Chen, C. Jang, S. Xiao, M. Ishigami, and M. S. Fuhrer, Nat. Nanotechnol. 3, 206 (2008).

[36] V. Perebeinos and P. Avouris, Phys. Rev. B 81, 195442 (2010).

[37] I.-T. Lin and J.-M. Liu, Appl. Phys. Lett. 103, 081606 (2013).

[38] Z.-W. Wang, L. Liu, and Z.-Q. Li, Appl. Phys. Lett. 106, 101601 (2015).
[39] E. H. Hwang, R. Sensarma, and S. Das Sarma, Phys. Rev. B 82, 195406 (2010).

[40] W. Xiaoguang, F. M. Peeters, and J. T. Devreese, Phys. Rev. B 32, 6982(R) (1985).

[41] E. H. Hwang and S. Das Sarma, Phys. Rev. B 87, 115432 (2013).

[42] K. Kaasbjerg, K. S. Thygesen, and K. W. Jacobsen, Phys. Rev. B 85, 115317 (2012).

[43] Z.-Y. Ong and M. V. Fischetti, Phys. Rev. B 86, 165422 (2012).

[44] R. Jalabert and S. Das Sarma, Phys. Rev. B 40, 9723 (1989).

[45] V. Perebeinos, S. V. Rotkin, A. G. Petrov, and P. Avouris, Nano Lett. 9, 312 (2008).

[46] S. Ahn, E. H. Hwang, and H. Min, Phys. Rev. B 90, 245436 (2014).

[47] S. Fratini and F. Guinea, Phys. Rev. B 77, 195415 (2008).

[48] W. Zhao, Q. Wu, Q. Hao, J. Wang, M. Li, Y. Zhang, K. Bi, Y. Chen, and Z. Ni, Appl. Phys. Lett. 108, 131903 (2016).

[49] W.-K. Tse and S. Das Sarma, Phys. Rev. Lett. 99, 236802 (2007).

[50] S. Q. Wang and G. D. Mahan, Phys. Rev. B 6, 4517 (1972).

[51] C. Zhang and Y. Takahashi, J. Phys.: Condens. Matter 5, 5009 (1993).

[52] S. Sarma and B. Mason, Ann. Phys. 163, 78 (1985).

[53] T. Low, A. S. Rodin, A. Carvalho, Y. Jiang, H. Wang, F. Xia, and A. H. Castro Neto, Phys. Rev. B 90, 075434 (2014).

[54] A. S. Rodin and A. H. Castro Neto, Phys. Rev. B 91, 075422 (2015).

[55] T. Low, R. Roldán, H. Wang, F. Xia, P. Avouris, L. M. Moreno, and F. Guinea, Phys. Rev. Lett. 113, 106802 (2014).

[56] F. Jin, R. Roldán, M. I. Katsnelson, and S. Yuan, Phys. Rev. B 92, 115440 (2015). 